\title{
Experimental investigation of anti-colliding pulse mode-locked semiconductor lasers
}

\author{
Jun-Ping Zhuang, ${ }^{1,2, *}$ Vincenzo Pusino, ${ }^{1}$ Ying Ding, ${ }^{1}$ Sze-Chun Chan,,${ }^{2,3}$ and Marc Sorel ${ }^{1}$ \\ ${ }^{1}$ School of Engineering, University of Glasgow, Glasgow G12 8LT, UK \\ ${ }^{2}$ Department of Electronic Engineering, City University of Hong Kong, Hong Kong, China \\ ${ }^{3}$ State Key Laboratory of Millimeter Waves, City University of Hong Kong, Hong Kong, China \\ *Corresponding author: jzhuang7@cityu.edu.hk
}

Received October 22, 2014; revised December 2, 2014; accepted December 3, 2014; posted December 11, 2014 (Doc. ID 225431); published February 11, 2015

\begin{abstract}
We experimentally demonstrate anti-colliding pulse mode-locking (ACPML) in an integrated semiconductor laser. The device geometry consists of a gain section and a saturable absorber (SA) section located immediately next to one of the cavity facets. After depositing a low-reflection coating on the SA facet and a high-reflection coating on the gain section facet, the threshold is unchanged, while the modulation of the SA is increased. The data presented here confirm that the ACPML configuration improves the peak output power of the pulses, reduces the amplitude fluctuation and timing jitter, and expands the biasing parameter range over which the stable mode-locking operation occurs. () 2015 Optical Society of America

OCIS codes: (250.5960) Semiconductor lasers; (140.4050) Mode-locked lasers.

http://dx.doi.org/10.1364/OL.40.000617
\end{abstract}

Semiconductor mode-locked lasers offer a very compact and low-cost solution for short optical pulse generation at high repetition rates [1-5]. These lasers were investigated for a wide range of applications, including optical communications, optical sampling, and radio-over-fiber distribution [1,2]. The simplest mode-locking configuration is made by reverse-biasing a short section along the optical cavity. The short section acts as a saturable absorber (SA) [3-5]. However, under passive mode-locking operation, the generated pulse train is prone to high timing jitter and amplitude noise [6,7]. Stabilization of the mode-locked pulses was investigated using various approaches, such as referencing to microwave sources [8], continuous-wave optical injection [5], and optical feedback $[\underline{4}, \underline{5}, \underline{9}]$, at the expense of an increase in the complexity of the device. More compact geometries that have been shown to improve the stability of the pulse train include colliding-pulse mode-locking (CPML), in which the SA is placed in the middle of the cavity [10], and self-CPML (SCPML), in which the SA is asymmetrically placed at one end of the cavity near a high-reflection (HR) coating [11].

Recently, Javaloyes and Balle analyzed an alternative configuration termed anti-colliding pulse mode-locking (ACPML) [12]. The ACPML geometry consists of a standard semiconductor laser geometry with a Fabry-Perot cavity formed between two cleaved facets with a forward-biased gain section and a short reverse-biased SA section. Unlike SCPML, the SA in the ACPML geometry is placed next to a facet with a low-reflection (LR) coating. To maintain an unchanged threshold current, the other facet is HR-coated to compensate for the extra cavity loss caused by the LR coating. As a result, the laser intensity builds up in the gain section as it travels from the HR-coated facet and impinges the SA with higher optical power $[12,13]$. The numerical analysis reveals improvements in timing jitter, amplitude noise, and in the extent of the mode-locking operation region, which is accompanied by an increase of the peak output power. Such an increase in peak power was also predicted for quantum-dot lasers with a similar coating geometry, although the numerical simulations on these devices indicate increased noise and the presence of unstable regimes [14].

In this Letter, we report on an experimental evaluation of the mode-locking performance in an ACPML configuration. The data provide the first confirmation of the theoretical predictions of the simultaneous improvements in peak pulse power, pulse amplitude noise, timing jitter, and in the extent of the mode-locking region. Interestingly, there is a concurrent experiment that contrasts ACPML with SCPML [15]. This experiment focuses on microwave power and linewidth.

The mode-locked laser in this work was fabricated in a multi-quantum well (MQW) InAlGaAs/InP epitaxial structure [16]. It was cleaved to a total length of $1.25 \mathrm{~mm}$, which corresponds to a repetition frequency of about $35 \mathrm{GHz}[3,17,18]$. The SA section constitutes $3 \%$ of the total cavity length. The SA is positioned immediately next to one of the facets of the laser and is electrically isolated from the gain section by a $10 \mu \mathrm{m}$ gap in the metallization layer [see Fig. 1(a)]. The laser is initially left uncoated so

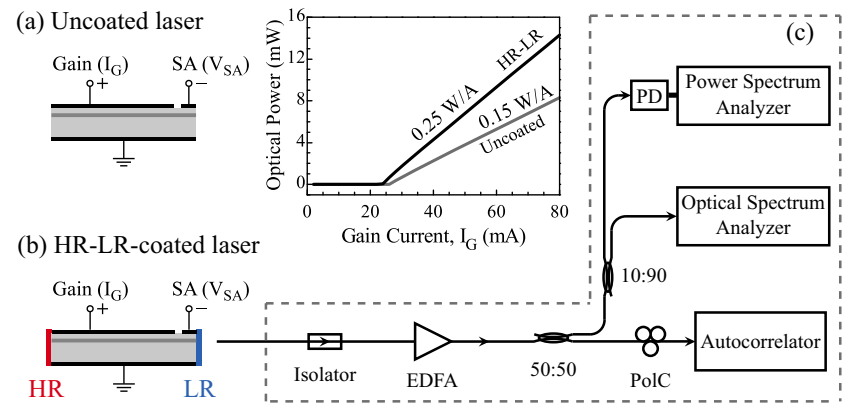

Fig. 1. (a) Original uncoated laser. (b) HR-LR-coated laser for ACPML. (c) Setup for mode-locked pulses characterization. PolC, polarization controller; PD, photodetector. Inset: Average optical output power versus gain section current for the uncoated laser (gray) and HR-LR-coated laser (black) when the SA is unbiased. 
that the cavity is formed by the cleaved facets with reflectivities of $30 \%$. The performances of the device are evaluated as a function of the forward biasing on the gain section $I_{G}$ and the reverse biasing on the SA section $V_{\mathrm{SA}}$. The gain section and SA facets of the same device are then coated with HR and LR, respectively, to realize the ACPML configuration [Fig. 1(b)]. On the SA section facet, RF sputtering is used to deposit a $200 \mathrm{~nm}$ thick layer of $\mathrm{SiO}_{2}$ and to realize the $\mathrm{LR}$ coating. This layer reduces the reflectivity to about $10 \%$, which is confirmed by the evaluation of the spontaneous emission spectra using the Hakki-Paoli technique [19]. On the gain section facet, a layer of $\mathrm{SiO}_{2}$ is first deposited for the purposes of electrical isolation from the gain section electrodes. This is followed by the deposition of $10 \mathrm{~nm}$ of $\mathrm{Ti}$ and $60 \mathrm{~nm}$ of $\mathrm{Au}$. These metal layers provide a reflectivity of over $90 \%$.

The inset of Fig. 1 shows the time-averaged optical output power from the SA facet as a function of $I_{G}$, with the SA left floating. The laser is kept at $20^{\circ} \mathrm{C}$ throughout all of the measurements. The gray curve is the output power measured from the original laser before the coatings were deposited [Fig. 1(a)], while the black curve is the power measured from the same laser after the HR and LR coatings were deposited [Fig. 1(b)]. The curves indicate a nearly-unchanged threshold current at around $25 \mathrm{~mA}$. Nonetheless, the HR-LR-coated laser has a slope efficiency of $0.25 \mathrm{~W} / \mathrm{A}$. This is an enhancement of 1.7 times that of the uncoated laser, due to the increased output coupling through the LR facet. The enhancement is slightly less than the ideal value of 2 , due to the non-ideal HR coating and internal gain dynamics $[12,13]$.

The mode-locked pulses from the configurations in Figs. 1(a) and 1(b) are characterized using the setup in Fig. 1(c). The optical pulses emitted from the SA facet are coupled into a lensed fiber, passed through an optical isolator, and amplified by an erbium-doped fiber amplifier (EDFA; PriTel SPFA-22), which is dispersioncompensated to avoid pulse broadening. Then, the optical pulses are distributed through 50:50 and 10:90 fiber couplers to an autocorrelator (Femotochrome Research FR-103XL), an optical spectrum analyzer (Advantest Q8384), and a $50 \mathrm{GHz}$ photodetector (PD; u2t Photonics XPDV2020R), which is followed by a power spectrum analyzer (Rohde \& Schwarz FSV40).

Figure 2 shows the experimental data in gray and black for the original uncoated and for the HR-LR-coated laser, respectively. The laser is kept biased at $\left(V_{\mathrm{SA}}, I_{G}\right)=$ $(-2.4 \mathrm{~V}, 72 \mathrm{~mA})$, which is the operating point for the uncoated laser that delivers the narrowest autocorrelation trace. Figure 2(a) records the second harmonic intensity output from the autocorrelator. The red curves in Fig. 2(a) illustrate the fitting of the autocorrelation trace by considering sech ${ }^{2}$ mode-locked pulses $[17,20]$. The fitting yields the autocorrelation's full width at half-maximum (FWHM), which is represented by $\Delta \tau=0.94$ to $1.04 \mathrm{ps}$. This deconvolves to optical pulsewidths between 0.61 and $0.67 \mathrm{ps}$. The pulse peak power is estimated by dividing the averaged output power by the pulsewidth and the repetition frequency. The HR-LR coatings are found to increase the peak power by 1.6 times to $0.17 \mathrm{~W}$.

Figure 2(b) shows the optical spectrum of the laser emission. The peak wavelength indicated is significantly
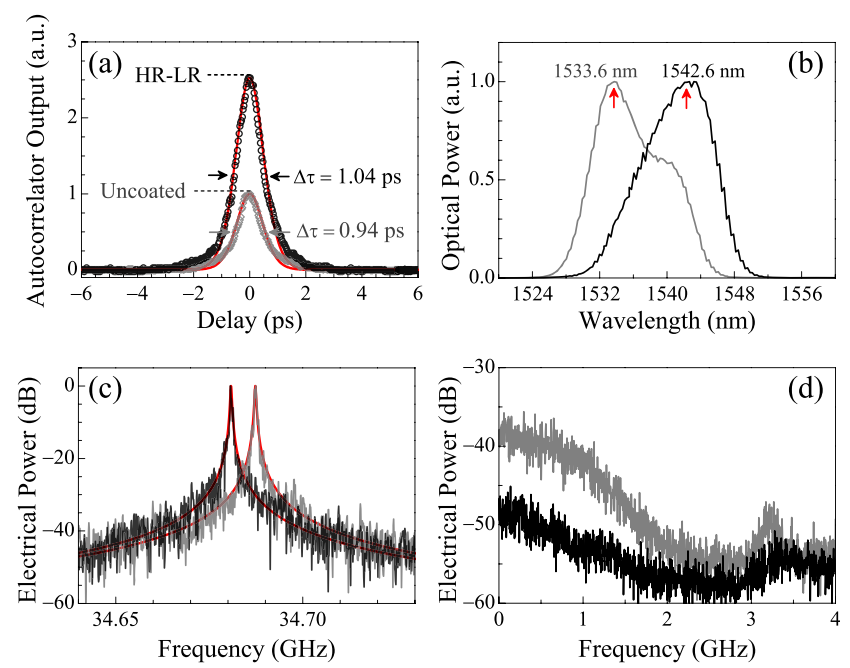

Fig. 2. (a) Autocorrelation pulse trace, (b) optical spectrum, (c) power spectrum at the mode-locking repetition frequency, and (d) power spectrum at the baseband. The power spectra are normalized to their respective peaks at the repetition frequency. Gray, original uncoated laser. Black, HR-LR-coated laser.

red-shifted from $1533.6 \mathrm{~nm}$ for the uncoated laser to $1542.6 \mathrm{~nm}$ for the HR-LR-coated device. The spectral shape is also modified by the presence of the coatings. The causes that determine such a pronounced change in the spectral behavior are not fully understood and are currently being investigated in more detail. In Fig. 2(c), the power spectrum normalized to the peak is shown as being around the repetition frequency, near $34.7 \mathrm{GHz}$. The RF peak in the ACPML configuration is just slightly shifted by about $10 \mathrm{MHz}$ downward while the FWHM is maintained at about $430 \mathrm{kHz}$, according to Lorentzian fitting. Despite showing few changes in the power spectrum around the repetition frequency, the amplitude of the power spectrum is greatly suppressed at low frequencies. Figure 2(d) shows the baseband power spectrum, where the magnitude is normalized to the spectral peak at the repetition frequency. The baseband spectrum corresponds to the pulse amplitude fluctuation of the mode-locked pulses [21] and is also related to the self-pulsation instabilities [22]. By integrating the baseband spectrum of Fig. 2(d) as a measure of the amplitude noise power, the HR-LR coatings are found to significantly suppress the amplitude noise by as much as $8 \mathrm{~dB}$.

The data of Fig. $\underline{2}$ are taken for a single set of biasing parameters and therefore only provide limited information on mode-locking behavior. Figures 3 and $\underline{4}$ show the characterization of the mode-locked pulses over a range of $V_{\mathrm{SA}}$ to $I_{G}$. This enables us to thoroughly compare the performance of the laser both without coating and with HR-LR coatings. The results obtained from the uncoated and the HR-LR-coated lasers are shown in the left and right columns, respectively. To limit the attention to mode-locking operation, only the operation region with amplitude noise below $0 \mathrm{~dB}$ and autocorrelation FWHM $\Delta \tau<2.5$ ps are shown. The contour lines of the autocorrelation FWHM $\Delta \tau$ in Fig. 3(a) indicate that only small changes occur in the pulsewidth as a function of the biasing parameters. The minimum values remain at around $0.94 \mathrm{ps}$ in both configurations. The HR-LR 

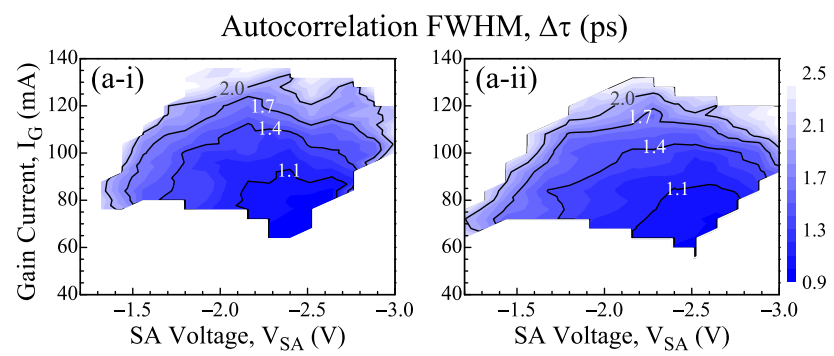

Pulse Peak Power (W)
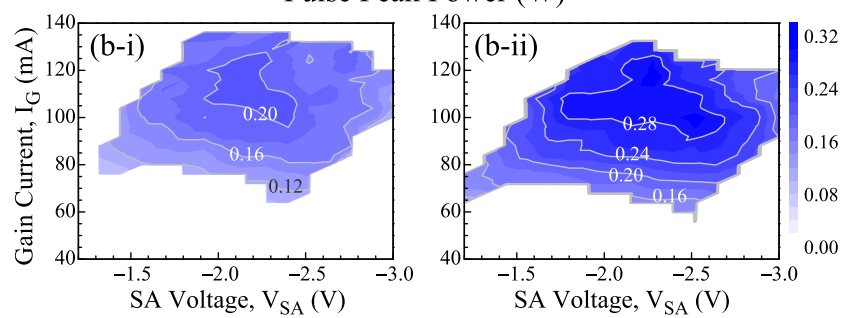

Peak Wavelength (nm)
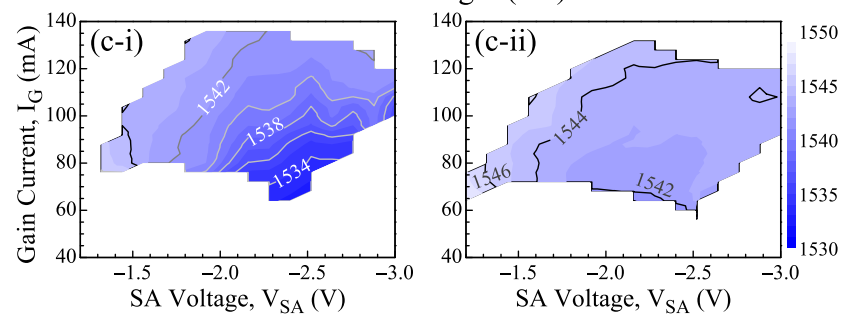

Time-bandwidth Product (TBP)
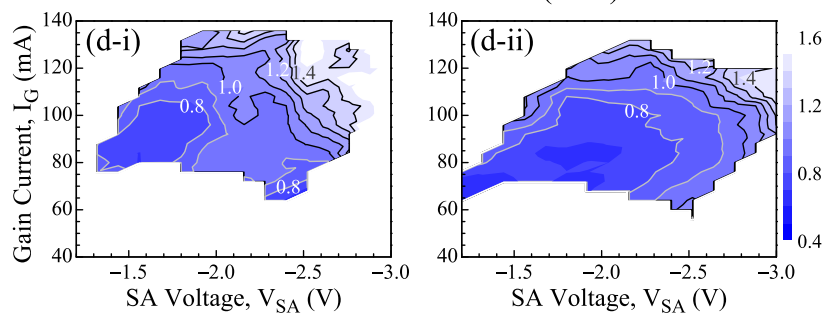

Fig. 3. (a) Autocorrelation FWHM $\Delta \tau$, (b) estimated peak power, (c) emission peak wavelength, and (d) TBP for the original uncoated laser (left column) and the HR-LR-coated laser (right column). Only mode-locked regions with amplitude noise below $0 \mathrm{~dB}$ and $\Delta \tau<2.5 \mathrm{ps}$ are shown.

coatings cause no or little reduction in pulsewidth, which confirms previous simulation results [12]. The contour maps of the estimated peak pulse power are shown in Fig. 3(b). The maximal peak power increases from 0.22 to $0.32 \mathrm{~W}$ in the ACPML configuration. The maps of the peak wavelength in Fig. 3(c) reveal a general red-shifting of the peak wavelength as $I_{G}$ increases. Of great interest is that the peak wavelength becomes less sensitive to the biasing conditions in the ACPML configuration. In fact, the peak wavelength in the uncoated device shifts as much as $12 \mathrm{~nm}$ over the whole modelocking region, while it shifts less than $5 \mathrm{~nm}$ in the coated device. Finally, Fig. 3(d) shows that the time-bandwidth product (TBP) is reduced from a minimum value of 0.70 to 0.66 . Similar to the behavior measured for the peak wavelength, the TBP also shows a pronounced enlargement of the biasing region over which values are low $(\mathrm{TBP}<0.8)$ and do not significantly vary.

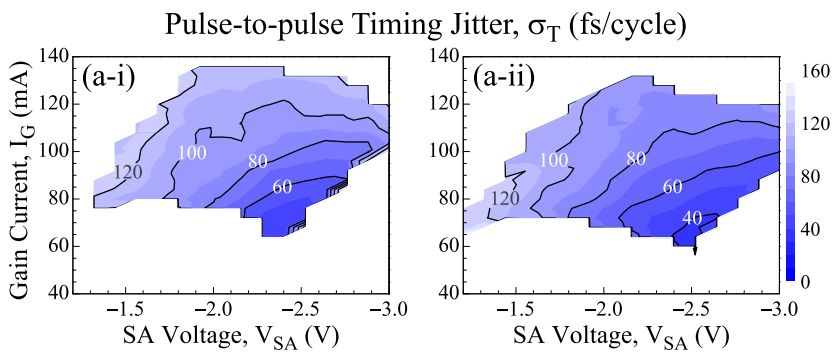

Relative Amplitude Noise Power (dB)
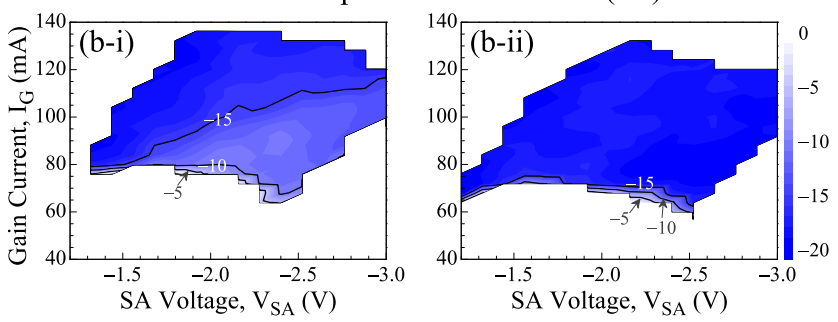

Fig. 4. (a) Pulse-to-pulse timing jitter $\sigma_{T}$ and (b) relative amplitude noise power for the original uncoated laser (left column) and the HR-LR-coated laser (right column). Only mode-locked regions with amplitude noise below $0 \mathrm{~dB}$ and $\Delta \tau<2.5$ ps are shown.

To quantify the timing fluctuations, the pulse-to-pulse root-mean-square timing jitter $\sigma_{T}$ is evaluated from the FWHM linewidth of the fundamental RF signal at the repetition frequency [6]. The data in Fig. 4(a) indicate that the minimal $\sigma_{T}$ reduces from 40 to $33 \mathrm{fs} / \mathrm{cycle}$, and that the low-jitter region of $\sigma_{T}<60 \mathrm{fs} /$ cycle expands in the ACPML configuration. To quantify the amplitude fluctuations, the relative amplitude noise power is calculated by integrating the power spectrum from $1 \mathrm{MHz}$ to $4 \mathrm{GHz}$. Here, the power spectrum is normalized to the spectral peak at the repetition frequency [21]. From Fig. 4(b), it is clear that the region with amplitude noise lower than $-15 \mathrm{~dB}$ is also substantially enlarged. Therefore, the stability of the mode-locked pulses is improved, both in terms of timing jitter and amplitude noise, which further confirms the benefits of the ACPML cavity design. Furthermore, upon observing Fig. 4(a-ii) in detail, it is revealed that $\sigma_{T}$ increases with $I_{G}$ in most parts of the map shown. Such behavior is consistent with an experiment on the microwave linewidth [15] as well as the theoretical prediction of high-bias currents [12].

In summary, the impact of anti-colliding cavity design on the performance of a two-section passively mode-locked laser is experimentally investigated. By introducing an LR coating to the facet near the SA and an HR coating to the other facet, the peak pulse power increases 1.6 times, the pulse amplitude noise is suppressed by $8 \mathrm{~dB}$, and the minimum timing jitter is reduced from 40 to 33 fs/cycle. Over the region of mode-locked operation, the minimum pulsewidth is maintained at about $0.67 \mathrm{ps}$, and the minimum TBP is slightly reduced from 0.70 to 0.66 . A large improvement offered by the ACPML configuration is that the operating regions show low jitter, and low TBP is substantially broadened. In addition, the emission peak wavelength is red-shifted and becomes much less sensitive to the biasing conditions. These features are of particular relevance for enhancing the long-term stability of the device and increasing the 
robustness against parameter fluctuations, such as current and temperature.

The authors would like to thank Salvador Balle and Julien Javaloyes for fruitful discussions and the technical staff of the James Watt Nanofabrication Centre at Glasgow University for supporting the device fabrication. This work was supported by the Royal Society under Project IE120157, the Glasgow Research Partnership in Engineering Programme and the Engineering and Physical Sciences Research Council under Project EP/ P504937/1, and the Research Grant Council of Hong Kong under Project CityU 110712.

\section{References}

1. K. Williams, M. Thompson, and I. White, New J. Phys. 6, 179 (2004).

2. E. Rafailov, M. Cataluna, and W. Sibbett, Nat. Photonics 1, 395 (2007).

3. L. Hou, P. Stolarz, J. Javaloyes, R. P. Green, C. N. Ironside, M. Sorel, and A. C. Bryce, IEEE Photon. Technol. Lett. 21, 1731 (2009).

4. C. Y. Lin, F. Grillot, Y. Li, R. Raghunathan, and L. F. Lester, IEEE J. Sel. Topics Quantum Electron. 17, 1311 (2011).

5. E. Sooudi, C. de Dios Fernandez, J. McInerney, G. Huyet, F. Lelarge, K. Merghem, R. Rosales, A. Martinez, A. Ramdane, and S. Hegarty, IEEE J. Sel. Top. Quantum Electron. 19, 1101208 (2013).

6. F. Kéfélian, S. O’Donoghue, M. T. Todaro, J. G. McInerney, and G. Huyet, IEEE Photon. Technol. Lett. 20, 1405 (2008).

7. C. Y. Lin, F. Grillot, Y. Li, R. Raghunathan, and L. F. Lester, Opt. Express 18, 21932 (2010).
8. R. Arkhipov, A. Pimenov, M. Radziunas, D. Rachinskii, A. G. Vladimirov, D. Arsenijevic, H. Schmeckebier, and D. Bimberg, IEEE J. Sel. Top. Quantum Electron. 19, 1100208 (2013).

9. M. Haji, L. Hou, A. E. Kelly, J. Akbar, J. H. Marsh, J. M. Arnold, and C. N. Ironside, Opt. Express 20, 3268 (2012).

10. Y. K. Chen and M. Wu, IEEE J. Quantum Electron. 28, 2176 (1992).

11. D. Jones, L. Zhang, J. Carroll, and D. Marcenac, IEEE J. Quantum Electron. 31, 1051 (1995).

12. J. Javaloyes and S. Balle, Opt. Lett. 36, 4407 (2011).

13. J. Javaloyes and S. Balle, IEEE J. Quantum Electron. 46, 1023 (2010).

14. H. Simos, M. Rossetti, C. Simos, C. Mesaritakis, T. Xu, P. Bardella, I. Montrosset, and D. Syvridis, IEEE J. Quantum Electron. 49, 3 (2013).

15. V. Moskalenko, V. Anand, M. Smit, K. Williams, and E. Bente, in Benelux Chapter Symp. Proc. (IEEE Photonics Society, 2014), pp. 115-118.

16. L. Hou, M. Haji, J. H. Marsh, and A. C. Bryce, Opt. Express 19, B75 (2011).

17. L. Hou, M. Haji, J. Akbar, B. Qiu, and A. C. Bryce, Opt. Lett. 36, 966 (2011).

18. A. D. Simard, M. J. Strain, V. Pusino, M. Sorel, and S. LaRochelle, Opt. Express 22, 17050 (2014).

19. B. W. Hakki and T. L. Paoli, J. Appl. Phys. 46, 1299 (1975).

20. K. Yvind, D. Larsson, L. Christiansen, C. Angelo, L. Oxenlowe, J. Mork, D. Birkedal, J. Hvam, and J. Hanberg, IEEE Photon. Technol. Lett. 16, 975 (2004).

21. D. von der Linde, Appl. Phys. B 39, 201 (1986).

22. M. J. Strain, M. Zanola, G. Mezösi, and M. Sorel, Opt. Lett. 37, 4732 (2012). 